\title{
Development of doubled haploid maize lines by using in vivo haploid technique
}

\author{
Rahime Cengiz $^{1 *}\left(\mathbb{D}\right.$, Kayıhan Zahit Korkut ${ }^{2}$ (D)
}

${ }^{1}$ Sakarya University of Applied Sciences, Field Crops Department, 54050 Serdivan, Sakarya, Turkey.

${ }^{2}$ Tekirdağ Namık Kemal University, Field Crops Department, 59030 Süleymanpaşa, Tekirdağ, Turkey.

\section{Article History}

Received 08 March 2020

Accepted 13 April 2020

First Online 20 April 2020

\section{* Corresponding Author}

Tel.: +905559670353

E-mail: rcengiz24@gmail.com

\section{Keywords \\ Breeding \\ Chromosome doubling \\ Doubled haploid \\ In vivo maternal haploid \\ Maize}

\begin{abstract}
The doubled haploid technology is now an integral component of modern maize breeding programs. In this study, the maternal haploid induction (gynogenesis) method was used to derive Doubled-Haploid (DH) lines from elite maize germplasm adapted to Turkey. Temperate haploid inducers (RWS, RWK-76, RWS x RWK-76 and WS14) were used as pollinators, and a set of 30 single-crossses (in FAO 650-700 maturity groups) were used as source materials. Putative haploid seeds were selected based on expression of R1-nj anthocyanin color marker. Highest haploid induction rate (20.42\%) was recorded by using RWK-76 as inducer line, and the lowest haploid induction rate $(17.75 \%)$ was obtained through WS14. Putative haploid seeds were germinated and seedlings were treated with $0.06 \%$ colchicine $+0.5 \%$ dimethylsulfoxide solution. Following transfer of seedlings into the field, $2178 D_{0}$ plants were obtained out of a total of 3012 treated haploids. Live plants were from $89 \%$ of 2178 seedlings which are planted to the field. Fertile plants were formed $57 \%$ of live plants. Inbreeding was succeeded in $31.23 \%$ of fertile plants and only $7.8 \%$ of inbreeding plants were able to produce seeds. Consequently, 27 doubled haploid lines were developed.
\end{abstract}

\section{Introduction}

Obtaining of inbred lines is the first and basic step in hybrid maize breeding. The aim of self-pollination is to obtain homozygous lines to be used as parents in developing the hybrid variety. At least seven generations of selfing is required to obtain an inbred line with $99 \%$ of homozygosity. Obtaining inbred lines is the most time-consuming part of the hybrid maize variety development process. New alternative complementary and supporting methods can be used to shorten the conventional hybrid maize breeding program (Cengiz et al., 2013).

Chase (1951) demonstrated the practical application of $\mathrm{DH}$ in maize breeding. In vivo haploid induction is now widely used in maize research and breeding. DH mapping populations are used to analyze linkage disequilibrium and analysis of haplotype-trait associations. In breeding, DH lines have enabled increased efficiency of line development and recurrent selection, and to reduce the effort for line maintenance (Röber et al., 2005).

The researchers stated that there are known two methods of in vivo haploid technique. Both are paternal haploid method which is used in order to obtain cytoplasmic male sterile form of elite line. Inducer line used in this technique carries the mutant indeterminate gametophyte recessive gene as female, unlike the inducer line used in the maternal haploid technique. Maternal haploid method is used for developing $\mathrm{DH}$ lines. Comparing the in vivo and in vitro haploid induction methods, the response of donor germplasm to the success of haploid achievement in the in vitro technique is very effective, whereas this success in the in vivo technique is less dependent on the donor 
response (Geiger 2009; Spitkó et al., 2006; Röber et al., 2005).

Geiger (2009) indicated that the most efficient haploid identification marker is the 'red crown' or 'navajo' kernel trait encoded by the dominant mutant allele R1-nj of the 'red color' gene $R 1$. There are different dominant genes such as $A 1, A 2$ and $C 2$ in the maize plant for coloration. The dominant gene $R 1-n j$ reveals deep coloration in the embryo and endosperm.

Using in vivo maternal haploid method, inducer lines are crossed to the donor germplasm not having the anthocyanin color markers at kernel, all the resulting hybrid kernels are expected to express the red color phenotype in the endosperm and in the scutellum as R1$n j$ is dominant over the colorless $r 1$ allele. Thus, the differential expression of R1-nj facilitates identification of maternal haploids from the diploid kernels. When maternal inducer lines with a high haploid induction rate (HIR) are used in the induction cross, putative haploids usually happen at a frequency of $6-10 \%$ (Chaikam \& Prasanna, 2012).

Gallais (1990) examined the effect of additive variance of germplasm to be used in obtaining $\mathrm{DH}$ lines. In this study, the covariance between the lines was calculated by evaluating the line value together with the additive variance. As a result, it was estimated that a $\mathrm{DH}$ line derived from the $F_{2}$ population combined about $50 \%$ better alleles than those derived from the $F_{1}$ population.

Prigge et al. (2011) have used hybrids, landraces and open-pollinated varieties as the source material for in vivo maternal haploid induction. Also, HIRs were compared between the dent and flint types. A significant variation was observed in terms of haploid induction in different source materials. Single-cross hybrids were found to have higher HIR than other source materials.

Hybrid maize breeding programs in Turkey have recently started using in vivo maternal haploid technique. This is the first study to report development of maternal haploids in Turkish maize germplasm using in vivo haploid induction. The aim of the study was to effectively use temperate haploid inducers in induction crosses hybrid maize varieties developed by Maize Research Institute, Sakarya, Turkey, and further develop $\mathrm{DH}$ lines using chromosome doubling of maternal haploids.

\section{Materials and Methods}

\section{Materials}

This study was conducted at Maize Research Institute, Sakarya, Turkey. A set of 30 elite single-cross hybrids (FAO 650-700 maturity group), were used as source materials. Of these 30 single-cross hybrids, 25 have dent kernel type and five had flint-like kernels.

The temperate haploid inducer lines RWS and RWK-76 and their cross RWS $\times$ RWK-76 were sourced from the University of Hohenheim, Germany, while the inducer line WS14 was obtained from French National Institute for Agricultural Research (INRA). The inducer genotypes used in this study had R1-nj allele (Figure 1) with anthocyanin color expression on the crown region of the endosperm and the embryo.

\section{Methods}

Inducer line was used as pollinators in in vivo maternal haploid technique. The source material was main donor and used as female parent. The source material used 30 single-cross hybrids were planted 3 rows. Tassels of source materials were removed by cutting on flowering period. Ears of source materials and tassels of inducer lines (50\% anthesis) were covered with papers. Every row of hybrid varieties was hybridized with each inducer line. Fertilized ears were kept in isolation papers until harvest. The names of the pollinators were written on isolation paper.

HIR was determined as below:

$\mathrm{HIR}=($ Number of haploids/Total number of seeds $) \times 100$

In vivo maternal haploid technique includes the following steps:

(I) Crossing the donors (e.g. individuals belonging to $F_{1}, F_{2}$ populations) as female parent with the inducer lines as male parent.

(II) Selection of putative haploid kernels (at the mature seed or root of seedlings) using the anthocyanin color markers.

(III) Germination of the putative haploid seeds.

(IV) Determination of effective and naturefriendly chromosome doubling agent and treatment to the putative haploid seedlings.

(V) Growing seedlings in optimum conditions and practice to necessary agronomic management

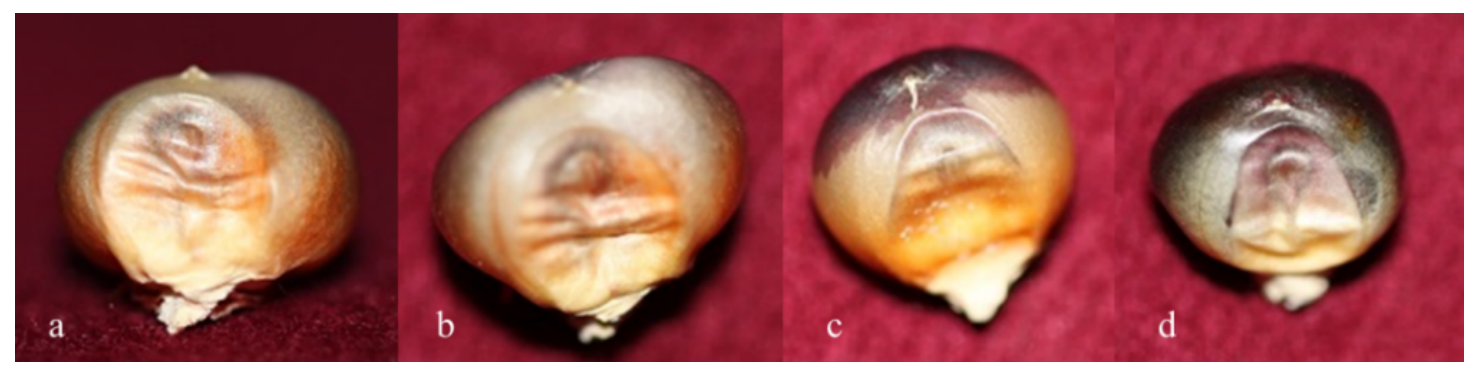

Figure 1. Expression of R1-nj color marker of inducer lines, (a) RWSxRWK-76, (b) RWK-76, (c) RWS, (d) WS14. 
of $D_{0}$ seedlings and obtain of $\mathrm{DH}$ seed by selffertilization $D_{0}$ plants.

(VI) Recurrent selection and application of $\mathrm{DH}$ lines in breeding programs.

Haploid kernels were identified using the anthocyanin color marker in the seeds obtained from induction cross (Figure 2).

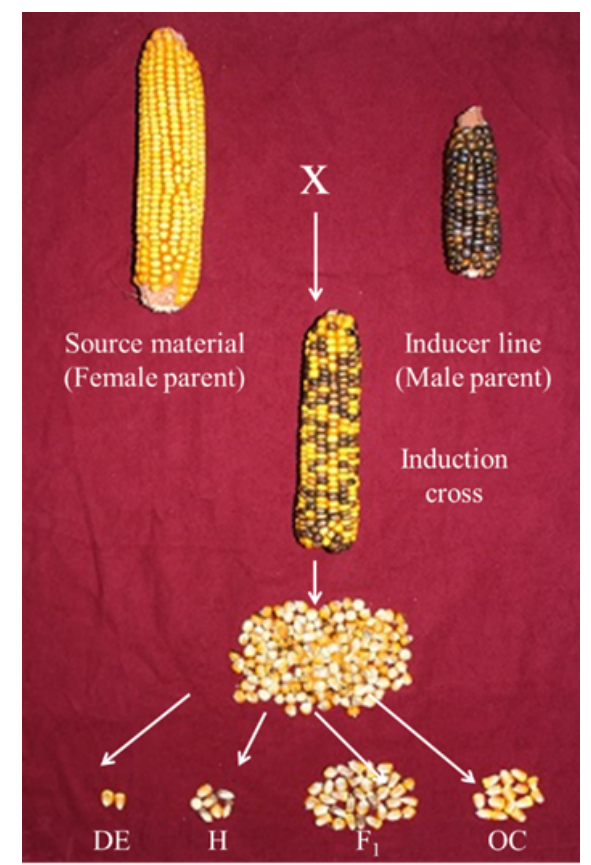

Figure 2. Haploid seed identification based on R1-nj marker expression. OC; outcross seeds, $F_{1}$; hybrid seeds with inducer line, $\mathrm{H}$; putative haploid seeds, DE; seeds with diploid endosperm.

Haploid seeds were germinated and treated the chromosome doubling agent at the coleoptile stage, 3 days after germination. Chromosome doubling protocol was followed based on Deimling et al. (1997). The whole seedlings were immersed into a $0.06 \%$ colchicine solution plus $0.5 \%$ dimethyl sulfoxide (DMSO) for $12 \mathrm{~h}$ at $20^{\circ} \mathrm{C}$. After the colchicine treatment, the seedlings were carefully washed in water and planted and grown in pots (during the first days under high humidity) in the growth chamber upto 3-4 leaf stage. A few weeks later the plants were transferred to field. Self-pollination of the $D_{0}$ plants were done.

\section{Results and Discussion}

Productivity pollen ability of RWS and RWK-76 lines are less than normal maize plants. Therefore, the number of seeds rate from obtained induction cross were low as against RWSxRWK-76. The number of pollinator plants of WS14 were high for producing seeds although ability of pollen production of WS14 was also low. A high number of plants increased seed formation rate as a result of induction cross.

Four different types of seeds were obtained from induction hybridization. In normal diploid or hybrid seeds, the endosperm and embryo had a purple color.
Although the putative haploid seeds and their endosperm had purple color, embryo of these seeds was colorless. On the other hand, in the seeds with diploid endosperm, the endosperm was colorless, while embryo was colorful. In putative outcross seeds, the embryo and endosperm were not colorful. The seeds obtained from the induction cross were divided according to these criterias given in Figure 3.

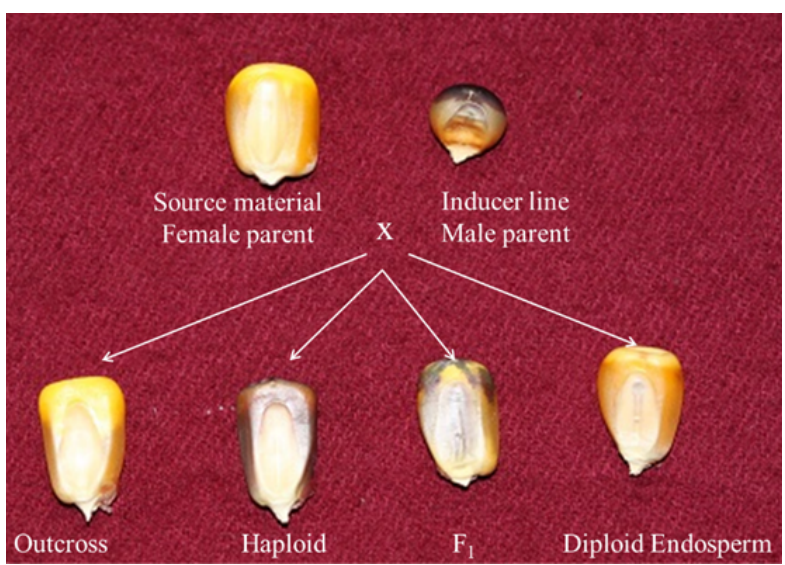

Figure 3. Identification of haploid seed based on R1-nj color marker.

The seeds obtained from induction hybridization with each inducer genotype were made distinction based on the four categories. Number of haploid seeds and the other categories on seeds obtained from induction hybridization were given on Table 1. HIR and rates of the other categories were given on Table 2 . The highest haploid induction rate is determined in RWK-76 inducer line although HIR of inducer genotypes were varied. According to the average of the studies carried out with many donors in different environments, RWS has an induction rate of about $8 \%$, RWK-76 recorded an average induction rate of $9-10 \%$, and the same rate was observed for the cross RWS $x$ RWK-76. WS14 had about 2-5\% haploid induction rate (Geiger 2009; Lashermes \& Beckert 1988). Most seeds belonged to $F_{1}$ category (54.14-68.01\%) and rate of seed with diploid endosperm was very low.

Chaikam and Prasanna (2012) indicated that R1-nj marker system offers an efficient way to determine haploids, but R1-nj expression could be highly influenced by the genetic background of the donor parent. The Navajo crown coloration might vary from a small spot (at the silk attachment point of the seed) to covering the entire aleurone (except the base). In addition, the intensity of color on the aleurone and scutellum may vary from very pale to deep. We found similar results to the study of these researchers (Figure 4). Differences in the occurrence of R1-nj may lead to misgrading. It is impossible to identify haploids when it is completely inhibited on both endosperm and embryo. All source germplasm in this study showed R1-nj expression on both endosperm and embryo. So, these dent and flint-like donor hybrids do not include inhibitor gene. 
Table.1. Number of haploid and the other categories of seeds as total number obtained from induction hybridization.

\begin{tabular}{ccccc}
\hline \multirow{2}{*}{$\begin{array}{c}\text { Inducer } \\
\text { genotypes }\end{array}$} & \multicolumn{5}{c}{ Seed number } \\
\cline { 2 - 5 } & Haploids & $\begin{array}{c}\text { Diploid } \\
\text { endosperm }\end{array}$ & $F_{1}$ & Outcross \\
\hline RWS x & 922 & 19 & 3215 & 571 \\
RWK-76 & 410 & 11 & 1256 & 392 \\
RWS & 413 & 15 & 1403 & 425 \\
RWK-76 & 473 & 25 & 3681 & 1886 \\
WS14 & 1207 & 70 & 9555 & 3274 \\
Total & 3012 & 15911 \\
\hline Grand total & \multicolumn{5}{c}{} \\
\hline
\end{tabular}

Table.2. HIR and the rates of the other categories calculated using total numbers of seeds obtained from induction hybridization.

\begin{tabular}{ccccc}
\hline Inducer & \multicolumn{5}{c}{$\%$} \\
\cline { 2 - 5 } genotypes & HIR & $\begin{array}{c}\text { Diploid } \\
\text { endosperm }\end{array}$ & $F_{1}$ & Outcross \\
\hline RWS $x$ & 19.50 & 0.40 & 68.01 & 12.08 \\
RWK-76 & 19.82 & 0.53 & 60.71 & 18.95 \\
RWS & 20.42 & 0.65 & 60.58 & 18.35 \\
RWK-76 & 17.75 & 0.37 & 54.14 & 27.74 \\
WS14 & 18.93 & & & \\
\hline $\begin{array}{c}\text { Average of } \\
\text { HIR }\end{array}$ & & & & \\
\hline
\end{tabular}

Some seeds were also lack of endosperm and embryo kernel formation as a result of haploid induction (Figure 5). Those seeds did not have germination ability and these numbers of seeds were not included in the other categories.

The precision and speed of putative haploid seed selection depend on the qualification of employees with good understanding of haploid identification through the R1-nj expression on aleurone and scutellum. According to the experiences, a light-flooded working environment and doing no more selection than four hours are critical.

Haploid identification is a lengthy process when there are a large number of seeds. Haploid identification can get difficult due to the limitations of R1-nj system. $B 1$ and PI1 system have also got limitation such as some germplasms also have purple root features (Rotarenco et al., 2010). Some researchers used original marker systems that could potentially facilitate automated haploid identification with the least false positives by using kernel oil content (Melchinger et al., 2014; Rotarenco et al., 2007). However, the source germplasm includes low oil content. Therefore, an automation system should be developed for the identification of different categories of seeds. These kinds of systems, like scanning the seeds as visually and by their content, would facilitate the identification of the haploid.

Altuntaş et al. (2018) used 87 haploid and 326 diploid maize seeds as their dataset. They used the expression of the R1-nj color marker in the embryo and endosperm. These features were obtained from gray

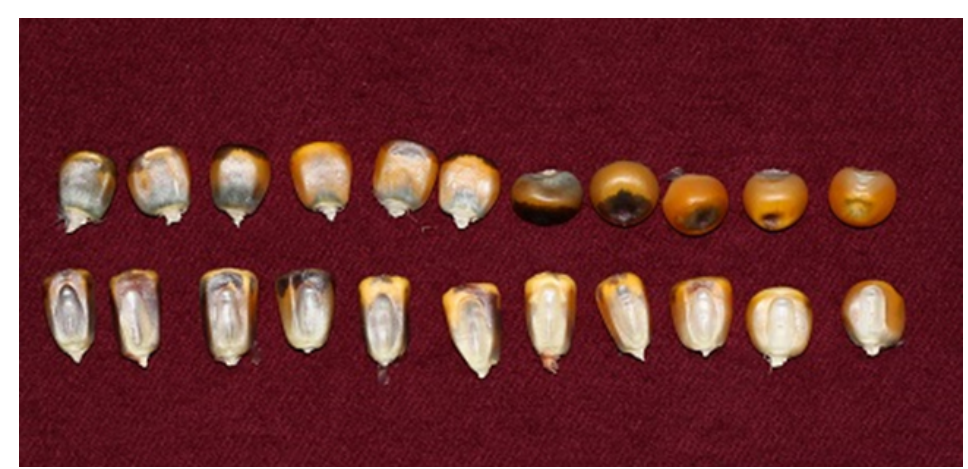

Figure 4. Showing distinctness in R1-nj expression on the aleurone and scutellum.

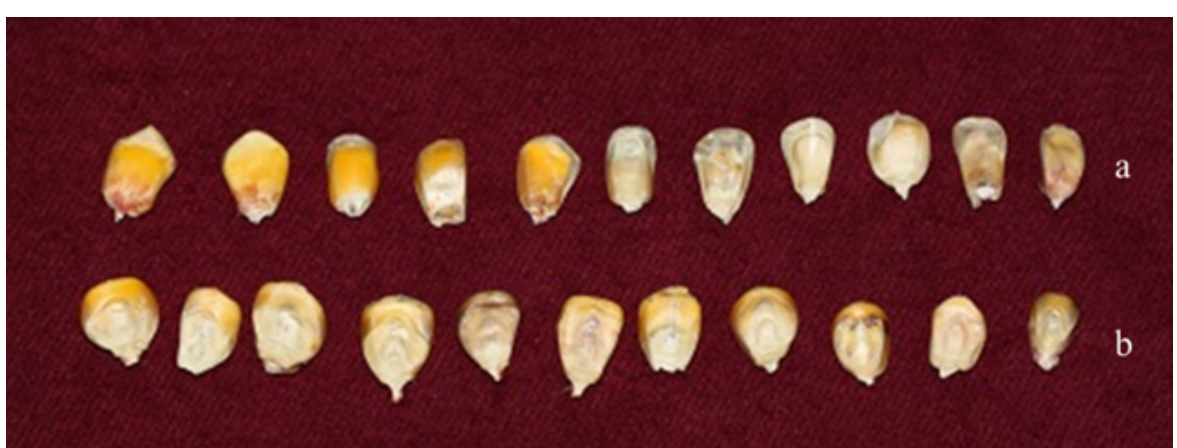

Figure 5. The seeds obtained from induction hybridization that were not included to the other categories, (a) without endosperm, (b) without embryo. 
level co-occurrence matrix. The feature vectors were classified using decision trees, k-nearest neighbors and artificial neural networks. The classification performance of machine learning tecniques was tested by using a 10 -fold cross-validation method. As a result of this test, the best performance was measured in decision tree with the classification success rate as $84.48 \%$.

Seeds with a haploid embryo include a regular triploid ( $3 n$ ) endosperm, for this reason, these seeds are adequate to show germination similar to those seeds with a diploid embryo (Coe \& Sarkar 1964). Haploid seeds were germinated in dark growth chamber at $23^{\circ} \mathrm{C}$ for 3 days. Putative haploid seedlings with a root length of $3 \mathrm{~cm}$ and coleoptile length of about $1.5-2 \mathrm{~cm}$ are ideal for colchicine application. Before colchicine application, root and coleoptile tissues were cut at about $2 \mathrm{~cm}$ and 1 $\mathrm{cm}$ from the tip, respectively, using a sterile scalpel blade or clippers. Cut seedlings that belong to the same single cross were kept in a mesh bag with its tag. Mesh bags with seedlings were kept in water till transferring to the colchicine tank.

Colchicine is the agent most commonly used to induce chromosome doubling. Colchicine disrupts mitosis by binding to tubulin, thus inhibiting the formation of microtubules and the polar migration of chromosomes, resulting in a cell with a doubled chromosome number (Barnabas et al., 1999). Chromosome doubling was applied in accordance with Deimling et al. (1997). After the colchicine application, the seedlings were planted in pots afterwards grown (during the 3 days under high humidity) in the growth chamber to the 3 to 4 leaf stage. $D_{0}$ seedlings were transplanted into the field manually. $D_{0}$ seedlings need less water than normal maize since they have weaker roots. For this reason, it is vital that the correct quantity of water is treated for good plant development. Maintenance of seedlings in the field was properly made such as fertilization application, weed control as manually and insect control. When $\mathrm{D}_{0}$ plants came into flowering stage, self-pollinating was made manually on $D_{0}$ plants and $\mathrm{DH}$ seeds were taken at harvest.

We observed that, after application of colchicine, all chromosomes of each cell in a seedling might not duplicate properly called sectoral diploidization. Some $D_{0}$ plants had tassels showing good production of pollen. Mostly $D_{0}$ plants had tassels producing small amounts of pollen or had anthers without pollen (Figure 6). Therefore, self-fertilization was forced to be done as repetitively.

DH ears were harvested by waiting for appropriate harvest moisture. Ears with red-colored seeds were discarded because they resulted from misclassified seed that could have been missed in the earlier steps. Frequently few seeds were set on the ears of DH plants (Figure 7).

Accordingly, we took utmost care to avoid seed loss in the field. The ears were carefully harvested by workers and kept in appropriate paper bags during transport to the storage for dehusking and drying. The seed representing the novel developed, entirely homozygous $\mathrm{DH}$ line will be sowed again for seed reproduction to be able to use the $\mathrm{DH}$ line in the future studies and breeding actions. The amount of seeds obtained from the first generation of $\mathrm{DH}$ lines are given in Figure 8.

\section{Conclusion}

In this study, $2178 D_{0}$ plants were obtained out of a total of 3012 treated haploids. The viability rate of 2178 seedlings transferred to the field was $89 \%$, and $57 \%$ of these surviving plants were fertile. Inbreeding was succeeded in $31.23 \%$ of fertile plants and only $7.8 \%$ of inbreeding plants were able to produce seeds. Consequently, $27 \mathrm{DH}$ lines were developed.

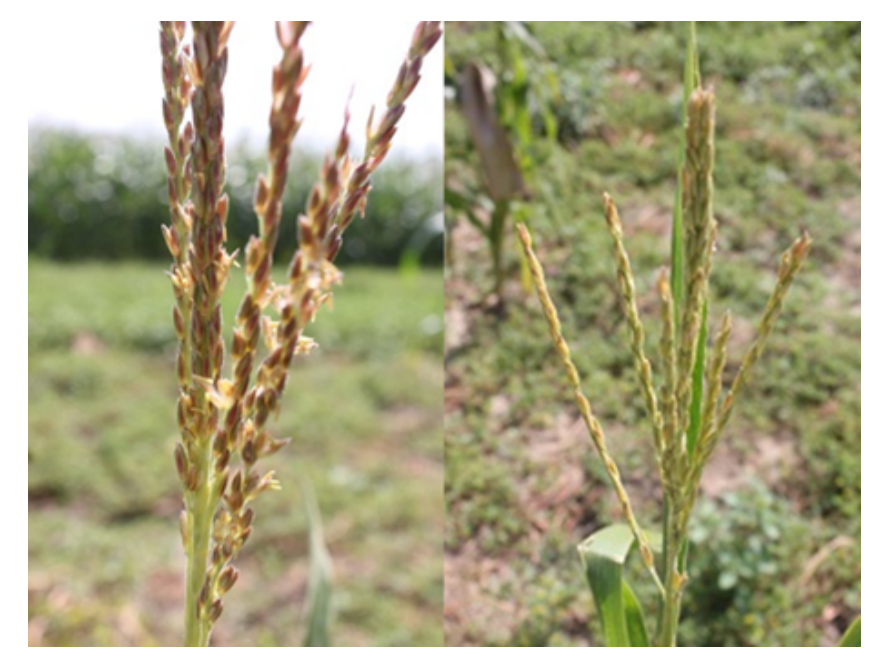

Figure 6. Tassel with a few branches are producing pollen which were sterile due to sectoral diploidization (left) and a completely sterile tassel (right). 


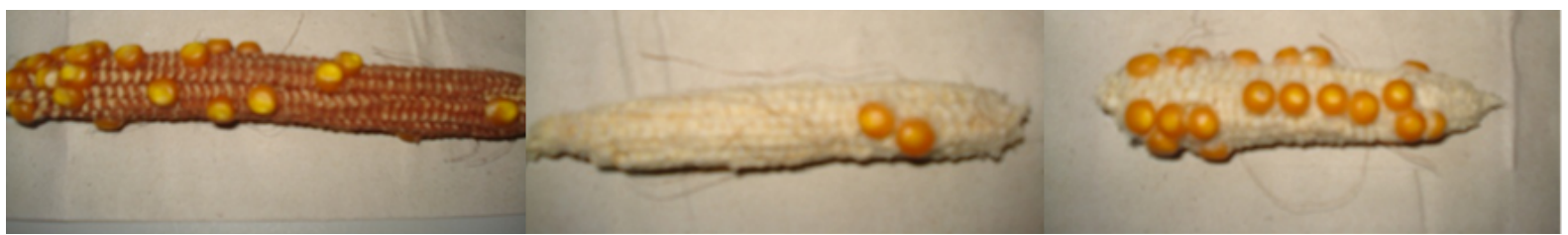

Figure 7. Some harvested ears of $D_{0}$.

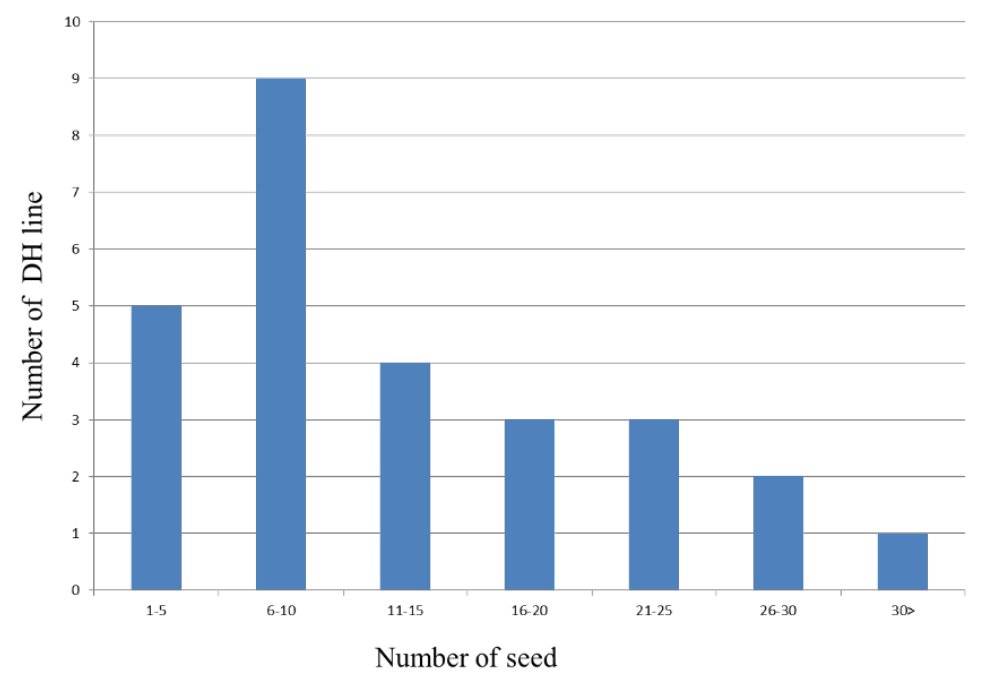

Figure 8. Amount of the seeds obtained from the first generation of $\mathrm{DH}$ lines.

Improvement of $\mathrm{DH}$ lines by in vivo maternal haploid induction needs specific abilities and equipments for i) great-quantity of chromosome doubling, ii) growing of putative haploid seedlings in the greenhouse, iii) transferring the seedlings to the field, iv) application of good growing conditions to seedlings, v) self-pollination of the $D_{0}$ plants.

In vivo maternal haploid technique has been an indispensable method of advanced breeding programs worldwide. Public research institutes and some private sector of carrying out maize breeding program in Turkey have started application in vivo maternal haploid technique in recent years.

\section{Acknowledgments}

This article was derived from Ph.D. Thesis of "Development of doubled haploid maize lines by using in vivo haploid technique" which was submitted to Tekirdağ Namık Kemal University in 2016. We are grateful to University of Hohenheim, Germany and French National Institute for Agricultural Research for providing RWS, RWK-76, RWS x RWK-76 and WS14 inducer lines and Maize Research Institute for offering opportunities.

\section{References}

Altuntaş, Y., Kocamaz, A. F., Cengiz, R. \& Esmeray, M. (2018). Classification of Haploid and Diploid Maize Seeds Using by Image Processing Techniques and Support Vector Machines, $201826^{\text {th }}$ Signal Processing and
Communications Applications Conference (SIU), İzmir.

Barnabás, B., Obert, B. \& Kovács, G. (1999). Colchicine, an efficient genome-doubling agent for maize (Zea mays L.) microspores cultured in anthero. Plant Cell Reports, 18, 858-862.

Cengiz, R., Cerit, İ., Tezel, M. \& Pamukçu, M. (2013). Kendilenmiş hatların elde edilmesi. In Melez Mısırla 100 Yıl Çalıştayı Kitabı (p. 115-136). Ankara: BISAB.

Chaikam, V. \& Prasanna, B. M. (2012). Maternal haploid detection using anthocyanin markers. In: Prasanna, B. M., Chaikam, V. \& Mahukued, G. (eds.), Doubled Haploid Technology in Maize Breeding: Theory and Practice (p. 21-24). CIMMYT, Mexico, DF.

Chase, S. S. (1951). Production of homozygous diploids of maize from monoploids. Agronomy, 44, 263-267.

Coe, E. H. \& Sarkar, K. R. (1964). The detection of haploids in maize. J. Heredity 55, 231-233.

Deimling, S., Röber, F. \& Geiger, H. H. (1997). Methodik und Genetik der in-vivo-Haploideninduktion bei Mais. Vortr Pflanzenzüchtung, 38, 203-224.

Gallais, A. (1990). Quantitative genetics of doubled haploid populations and application to the theory of line development. Genetics, 124, 199-206.

Geiger, H. H. (2009). Doubled haploids. In Bennetzen, J. L. \& Hake, S. (eds.), Handbook of Maize. Springer, New York, NY.

Lashermes, P. \& Beckert, M. (1988). Genetic control of maternal haploidy in maize (Zea mays L.) and selection of haploid inducing lines. Theoretical and Applied Genetics, 76, 405-410.

Melchinger, A. E., Schipprack, W., Friedrich, H. U. \& Mirdita, V. (2014). In vivo haploid induction in maize: identification of haploid seeds by their oil content. Crop Science, 54(4), 1497-1504.

Prigge, V., Sanchez, C., Dhillon, B. S., Schipprack, W., Araus, J. L., Banziger, M. \& Melchinger, A. E. (2011). Doubled haploids in tropical maize: I. Effects of inducers and 
source germplasm on in vivo haploid induction rates. Crop Science, 51, 1498-1506.

Rotarenco, V. A., Kirtoca, I. H. \& Jacota, A. G. (2007). Possibility to identify kernels with haploid embryo by oil content. Maize Genetics Cooperation Newsletter, 81(11).

Rotarenco, V. A., Dicu, G., State, D. \& Fuia, S. (2010). New inducers of maternal haploids in maize. Maize Genetics Cooperation Newsletter, 84, 15.
Röber, F. K., Gordillo, G. A. \& Geiger, H. H. (2005). In vivo haploid induction in maize-performance of new inducers and significance of doubled haploid lines in hybrid breeding. Maydica, 50, 275-283.

Spitkó, T., Sági, L., Pintér, J., Marton, L. C. \& Barnabás, B. (2006). Haploid regeneration aptitude maize (Zea mays L.) lines of various origin and of their hybrids. Maydica, 51, 537-542. 